\title{
Infection and Gene Expression of the Clubroot Pathogen Plasmodiophora brassicae in Resistant and Susceptible Canola Cultivars
}

\author{
Weixin Fei, College of Plant Protection, Anhui Agriculture University, Hefei, China; Anhui Academy of Agricultural Sciences, Hefei, China; \\ Jie Feng, Crop Diversification Centre North, Edmonton, AB, Canada; Songbai Rong, Anhui Academy of Agricultural Sciences, Hefei, China; \\ Stephen E. Strelkov, Department of Agricultural, Food and Nutritional Science, University of Alberta, Edmonton, AB, Canada; Zhimou Gao, \\ College of Plant Protection, Anhui Agriculture University, Hefei, China; and Sheau-Fang Hwang, Crop Diversification Centre North, \\ Edmonton, AB, Canada
}

\begin{abstract}
Fei, W., Feng, J., Rong, S., Strelkov, S. E., Gao, Z., and Hwang, S.-F. 2016. Infection and gene expression of the clubroot pathogen Plasmodiophora brassicae in resistant and susceptible canola cultivars. Plant Dis. 100:824-828.

Infection by the clubroot pathogen Plasmodiophora brassicae on resistant and susceptible canola cultivars was investigated at various times following inoculation. Primary infection occurred on more than $90 \%$ of root hairs in both cultivars at 7 days after inoculation (dai), and thereafter declined to less than $20 \%$ at 14 to 35 dai. The amount of primary infection on the two cultivars was similar at each time point. Secondary infections were rare in both cultivars at 5 and 7 dai but became common after 14 dai. At 14 to 28 dai, the level of secondary infection was greater in the resistant cultivar than in the susceptible one. The in planta

expression of 12 selected $P$. brassicae genes was investigated by reverse-transcription quantitative polymerase chain reaction. All genes were upregulated at 5 or 7 dai in the resistant cultivar. In the susceptible cultivar, the 12 genes could be classified into three groups according to their expression patterns: 2 genes showed an expression peak at 14 dai, 3 showed two expression peaks at 14 and 35 dai, and the others showed an expression peak at 35 dai. Results from this study will be useful in breeding for resistance and in selecting candidate pathogenicity genes for further studies.
\end{abstract}

Plasmodiophora brassicae Woronin causes clubroot disease on many species of the Brassicaceae family, and is an emerging threat to canola (Brassica napus) production in Canada (Hwang et al. 2012). As an obligate parasite, the pathogen has three distinct stages in its life cycle: survival in the soil as resting spores, primary infection of root hairs, and secondary infection of and development within the root cortex (Ingram and Tommerup 1972; Naiki and Dixon 1987). Each resting spore germinates to release one primary zoospore. These zoospores infect root hairs by penetrating the cell wall. Within the root hairs, primary plasmodia develop and cleave into zoosporangia, each containing 4 to 16 secondary zoospores, which are either released into the rhizosphere and then infect the root cortex from the outside or infect neighboring cortical cells directly from inside the root hair (Kageyama and Asano 2009). Secondary infection is followed by the development of secondary plasmodia within the root cortex, which eventually are cleaved into large numbers of resting spores within the clubbed roots. As the root tissues disintegrate, which is promoted by bacteria, the resting spores are released into the soil to complete the disease cycle.

Many strategies have been proposed for clubroot control (Hwang et al. 2014; Peng et al. 2014); among others, the use of resistant cultivars is believed to be most effective (Rahman et al. 2014). Several clubroot-resistant canola hybrids have been introduced into the $\mathrm{Ca}$ nadian market (Hwang et al. 2012). However, the molecular and biochemical mechanisms of the resistance in these cultivars are not fully understood. Moreover, genetic information on the pathogen was very limited until the recent release of its whole genome sequence

Corresponding authors: S.-F. Hwang; E-mail: sheau-fang.hwang@gov.ab.ca; and Z. Gao; E-mail: gaozhimou@126.com

*The $\boldsymbol{e}$-Xtra logo stands for "electronic extra" and indicates that two supplementary tables are published online.

Accepted for publication 28 November 2015

http://dx.doi.org/10.1094/PDIS-11-15-1255-RE

(C) 2016 The American Phytopathological Society
(Schwelm et al. 2015). A previous transcriptional study of 118 nonredundant $P$. brassicae genes (Feng et al. 2013a) indicated that most of these genes are differentially regulated between the stages of primary and secondary zoospores. However, none of these genes has been studied functionally.

In the current study, the infection processes of $P$. brassicae in resistant and susceptible canola cultivars were investigated and compared over the infection time course. Based on the cDNA derived from the infected plant samples collected during the time course, 12 genes were selected and subjected to a gene expression profile study by reverse-transcription quantitative polymerase chain reaction (RT-qPCR) analysis. The objectives of this study were to (i) clarify the infection stages restricted or prevented by the resistance in the tested cultivar and (ii) identify the $P$. brassicae genes up- or downregulated during the process of clubroot pathogenesis. Results obtained from this study will help to better understand the pathogenesis of clubroot, provide gene candidates for gene functional studies, facilitate breeding for resistance, and define potential targets for new pesticides.

\section{Materials and Methods}

The $P$. brassicae single-spore isolate, plant materials, and candidate genes. A single-spore isolate of $P$. brassicae, SACANss 1 , classified as pathotype 3 (Xue et al. 2008), was used to inoculate the plant materials. Pathotype 3 has been reported to be predominant in western Canada (Strelkov et al. 2006). The susceptible canola 'Westar' was used as host to maintain P. brassicae. Hybrid '45H26' (susceptible to pathotype 3) and '45H29' (resistant to pathotype 3) canola were used in the investigation of the in planta gene expression. Twelve $P$. brassicae genes were selected for analyses of their expression (Supplementary Table S1). These genes have been reported to be the most up- or downregulated in secondary zoospores compared with primary zoospores (Feng et al. 2013a). Sequences of these genes were retrieved from the National Center for Biotechnology Information database (http://www.ncbi. nlm.nih.gov) and a BLASTx search was conducted using all of the sequences as a batch. The GenBank accession numbers of these genes and the BLASTx best hits are listed in Supplementary Table S1. 
Collection of resting spores. Galls that developed on Westar after inoculation with SACAN-ss1 in a greenhouse were collected and kept at $-20^{\circ} \mathrm{C}$. Resting spores were collected from frozen galls and inoculum was prepared according to the procedures of Feng et al. (2013b). Briefly, galls were homogenized in $10 \%$ sucrose in a blender. The slurry was passed through eight layers of cheesecloth and the suspension was centrifuged at $50 \times g$ for $5 \mathrm{~min}$. In $10 \%$ sucrose solution, such a centrifugation would pellet most soil particles and large fragments of plant tissue. The supernatant was transferred to a new tube and centrifuged at $2,000 \times g$ for $5 \mathrm{~min}$. The resulting pellet consisted of two distinct layers: the lower black layer consisted of soil particles and the upper white to brownish layer of resting spores. The upper layer was resuspended in $5 \mathrm{ml}$ of water by gentle pipetting and transferred into a new tube containing $40 \mathrm{ml}$ of water. After centrifugation at $2,000 \times g$ for $5 \mathrm{~min}$, the supernatant was removed. To eliminate bacteria and other microorganisms associated with $P$. brassicae, resting spores in the pellet were adjusted to a concentration $1 \times 10^{8}$ resting spores $/ \mathrm{ml}$ and surface disinfected by incubating in colistin sulfate at $1 \mu \mathrm{g} / \mathrm{ml}$ and 1 vancomycin hydrochloride at $\mu \mathrm{g} / \mathrm{ml}$ at $25^{\circ} \mathrm{C}$ in the dark for $24 \mathrm{~h}$. The spores were used as inoculum after removal of the antibiotics by washing twice with $40 \mathrm{ml}$ of sterile water.

Inoculation. Canola seed were germinated on moist filter paper for 1 week at $24^{\circ} \mathrm{C}$ (day) and $18^{\circ} \mathrm{C}$ (night) with a 16-h photoperiod. Sunshine mix number 4 potting medium (SunGro Horticulture, Vancouver, BC, Canada) was inoculated with the resting spore suspension at a concentration of $1 \times 10^{6}$ spores $/ \mathrm{ml}$ of soil in a concrete mixer. The inoculated potting mix was placed in plastic boxes (21 by 34 by $11.8 \mathrm{~cm}$ ). The boxes were arranged on a bench in a randomized complete block design with the cultivars as treatment; each treatment consisted of three boxes as three replications. The seedlings were first inoculated by dipping the roots in a resting spore suspension $(1 \times$ $10^{7}$ spores $/ \mathrm{ml}$ ) for $20 \mathrm{~s}$, then transplanted into the inoculated soil at a density of 60 plants/box. The plants were kept in a greenhouse maintained at $24^{\circ} \mathrm{C}$ (day) and $18^{\circ} \mathrm{C}$ (night) with a 16-h photoperiod and watered from the bottom every second day with tap water at $\mathrm{pH} 6.4$ (adjusted with $\mathrm{HCl}$ ).

Sampling. Plants were sampled at 5, 7, 14, 21, 28, or 35 days after inoculation (dai), at which time 10 inoculated plants were randomly dug out from each box and the roots were washed with tap water. Seedlings that had been root dip inoculated but not transplanted were regarded as the 0-dai samples.

Microscopy investigation. At each time point except 0 dai, three $1-\mathrm{cm}$ fragments at the proximal end of the root from each of the three resistant or susceptible plants were examined to determine the extent of primary and secondary infections using a Zeiss AXIO microscope (Carl Zeiss, Oberkochen, Germany). On each root fragment, five fields of view using the $\times 20$ objective lens were examined. In each field of view, two sets of data were collected: number of root hairs with primary infection and the total number of root hairs, and the total number of secondary plasmodia. For either primary or secondary infections at each time point, the data from one replication (box) were averaged and the result was regarded as one data point for statistical analyses. This experiment was conducted twice, with similar results.

RNA extraction and RT-qPCR. At each time point, roots from three plants of the resistant or susceptible cultivar in each of the three boxes were ground in liquid nitrogen. From $750 \mathrm{mg}$ of the ground powder, total RNA was extracted using a PureYield RNA Midiprep kit (Promega Corp., Madison, WI). The extracted RNA was cleaned with a Qiagen RNase-Free DNase Set (Qiagen Canada, Toronto). The first-strand cDNA was synthesized from the resultant RNA using an iScrip cDNA Synthesis Kit (Bio-Rad, Hercules, CA) and subsequently purified with a QIAquick PCR Purification Kit (Qiagen Canada). Real-time PCR (qPCR) was conducted against the firststrand cDNA in a StepOnePlus Real-Time PCR System (Life Technologies, Carlsbad, CA) using the Power SYBR Green PCR Master Mix (Life Technologies). The qPCR was run in a $15-\mu 1$ reaction mixture containing $1.25 \mu \mathrm{l}$ each of $5 \mu \mathrm{M}$ forward and reverse primers and $50 \mathrm{ng}$ of the cDNA template. The comparative cycle threshold (CT) method was used to analyze and present the data on gene expression according to Schmittgen and Livak (2008). The P. brassicae Actinl gene (GenBank accession number AAR88383) was used to normalize the pathogen biomass. For each cDNA sample, the reactions were performed in triplicate. Primers for all qPCR analyses were synthesized by Integrated DNA Technologies (Coralville, IA) and their sequences are listed in Supplementary Table S2.

Data analysis. Data were analyzed using the Microsoft Excel addin DSAASTAT developed by Dr. Andrea Onofri at the University of Perugia, Italy (http://accounts.unipg.it/ onofri/index.htm). Analysis of variance (ANOVA) was carried out for each time point using the data on primary and secondary infections, as well as the in planta $P$. brassicae biomass assessed by the CT values of Actin1. The difference between the two cultivars was assessed by the $P$ values of an $F$ test. For the gene expression pattern, ANOVA were performed on the data from each gene in either cultivar; the differences among time points were assessed using the Fisher's least significant difference test $(P \leq 0.01)$.

\section{Results and Discussion}

Infection of resistant and susceptible cultivars. At each time point, similar levels of primary infection were observed in the resistant and susceptible cultivars (Fig. 1A). At 7 dai, almost all of the root hairs examined in both cultivars were infected (Fig. 1A). At and after 14 dai, however, primary infections declined to less than $20 \%$. Some root hairs regarded as uninfected may, indeed, have been infected, but the secondary zoospores had been released and the empty sporangia were degraded or became difficult to be distinguished from host tissue. However, considering the observation that most of the newly emerged root hairs were not infected, it is likely that primary infection was dependent on the development stage of the host plant because, otherwise, both the resting spores with postponed germination, which might only respond to contact with the root hairs (Feng et al. 2010), and the secondary zoospores released from primary infections (Feng et al. 2012, 2013b) should have been able to cause primary infections on the newly developed root hairs. Similarities in primary infection of resistant and susceptible cultivars of canola (Deora et al. 2012, 2013; Gludovacz et al. 2014) and of hosts and nonhosts (Feng et al. 2012) have been previously reported, which is in agreement with the observation from the current study. These data indicate that host specificity maybe not important for clubroot pathogenesis.

At 5 and 7 dai, secondary infections in the resistant and susceptible cultivars were at low but similar levels (Fig. 1B). At 14, 21, and 28 dai, levels of secondary infections increased in both cultivars and infections in the resistant cultivar occurred at higher levels than in the susceptible cultivar. Higher levels of secondary infection in the resistant compared with the susceptible cultivar were also observed in a previous study (Zhang et al. 2015), as well as in the current study when the entire time course was repeated. However, this observation was different from that of Deora et al. $(2012,2013)$ and Gludovacz et al. (2014), who reported that secondary infection was significantly less in resistant cultivars than in the susceptible cultivar. This may be due to the fact that different materials were used for secondary infection assessment: the proximal part of the root was used in the current study and the taproot $1 \mathrm{~cm}$ below the hypocotyl was used by Deora et al. (2012, 2013) and Gludovacz et al. (2014). In the susceptible cultivar, resting spores were observed under a microscope at 21 dai which, along with the visual evidence of gall formation, indicated that secondary plasmodia had started to develop into the resting spore stage thereafter. Secondary plasmodia accumulated in the resistant cultivar from 14 to 28 dai but were prevented or restricted by the resistance mechanism from further development. Despite the higher number of secondary plasmodia in the resistant cultivar versus the susceptible cultivar, the size of secondary plasmodia in the resistant cultivar was smaller than in the susceptible cultivar, based on visual assessment at and after 14 dai (Fig. 1C).

At 35 dai, a large number of resting spores were evident in the cortical cells in the clubbed roots (galls) of the susceptible cultivar (data not shown), and enlarged or fused secondary plasmodia were commonly observed in the unclubbed parts of the root (Fig. 1D). No galls 
or resting spores were observed on the resistant cultivar at any point during the time course, although they have been occasionally observed on this cultivar in other experiments (Hwang et al. 2013). At 35 dai, most of the secondary plasmodia were enlarged in comparison with those at 14 dai. On many of these plasmodia, an exit tube had formed through which the contents of the plasmodium escaped, leaving it empty (Fig. 1D). This is the first observation of such an exit tube on secondary plasmodia, although a similar structure has been reported on primary plasmodia (Buczacki and Clay 1984). It is likely that the empty secondary plasmodia are prone to be degraded in the plant cells, which partially explains the lower number of secondary plasmodia observed at 35 compared with 28 dai in the resistant cultivar (Fig. 1B).

A

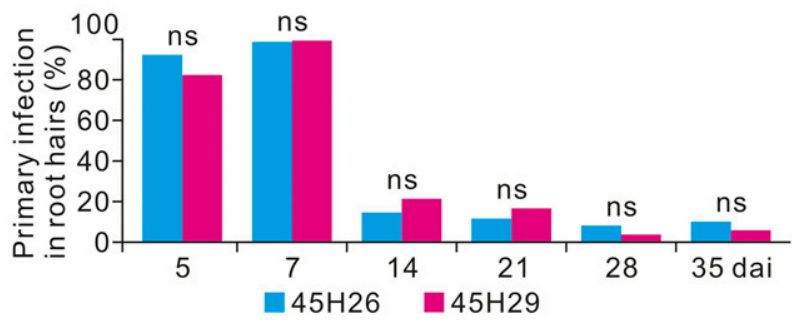

B

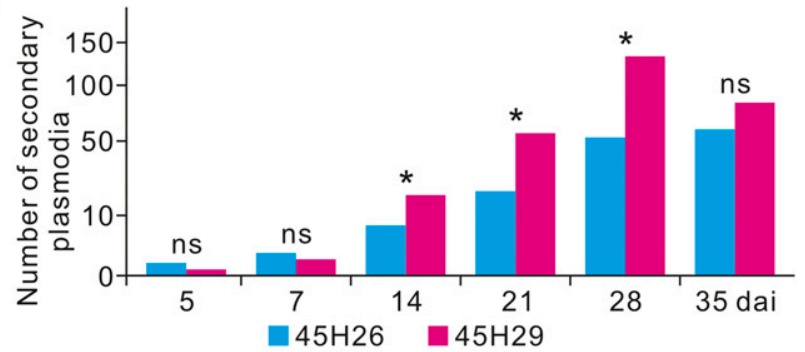

C

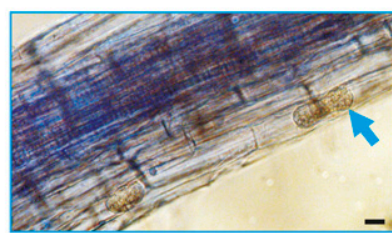

$45 \mathrm{H} 26$ (14 dai)

D

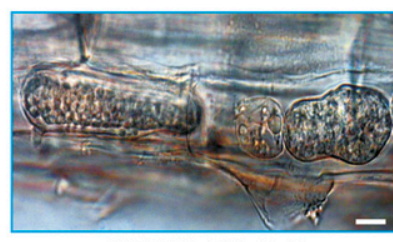

E

$45 \mathrm{H} 26$ (35 dai)

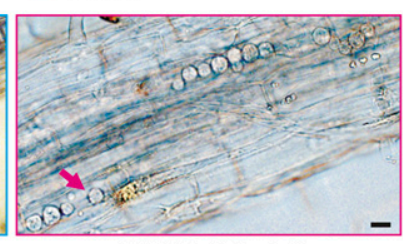

$45 \mathrm{H} 29$ (14 dai)

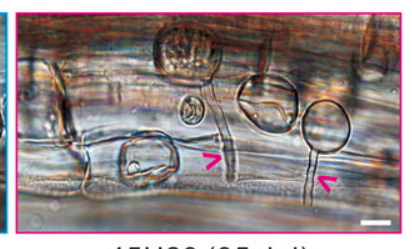

$45 \mathrm{H} 29$ (35 dai)

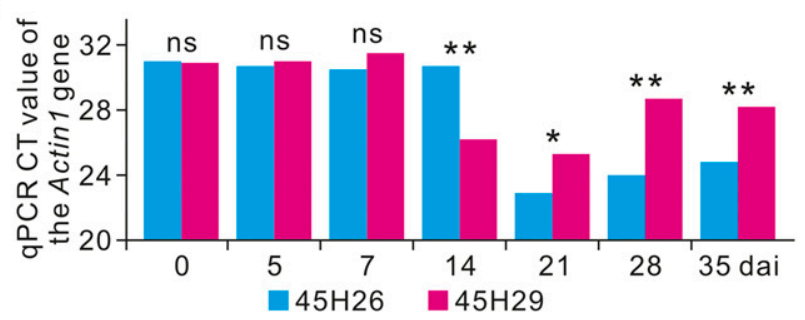

Fig. 1. Infection time course of the clubroot pathogen Plasmodiophora brassicae on susceptible canola $45 \mathrm{H} 26$ (left bar of each pair) and resistant canola $45 \mathrm{H} 29$ (right bar); ns, ${ }^{*}$, and ${ }^{* *}$ above bars indicate nonsignificant $(P>0.05)$, significant $(P \leq$ $0.05)$, and highly significant $(P \leq 0.01)$ differences, respectively, based on the $F$ test $(n=3)$. A, Primary infection; $\mathbf{B}$, secondary infection; and secondary plasmodia at C, 14 or D, 35 days after inoculation (dai). Arrows in $\mathbf{C}$ emphasize the size difference of plasmodia on the resistance and the susceptible cultivar. Arrowheads in $D$ indicate the exit tube through which the contents in the plasmodium escaped. Bars $=10 \mu \mathrm{m}$. E, Relative biomass of $P$. brassicae as evaluated by the cycle threshold (CT) value of the Actin1 gene.
The relative biomass of $P$. brassicae in the resistant and susceptible cultivars during the time course was compared by RT-qPCR based on 50-ng cDNA samples extracted from infected roots. The CT value of the Actin1 gene was used as the biomass indicator, which is inversely proportional to the amount of $P$. brassicae. At 0,5 , and 7 dai, $P$. brassicae biomass was similar in the resistant and the susceptible cultivars (Fig. 1E). At 14 dai, the CT value of Actin1 in the resistant cultivar was lower than in the susceptible cultivar, indicating that the biomass was higher in the resistant cultivar. This is consistent with the observation that more secondary plasmodia were observed at 14 dai in the resistant cultivar than in the susceptible cultivar but the secondary plasmodia in the susceptible cultivar occupied a larger space than secondary plasmodia in the resistant cultivar, because of their continuous enlargement and development. At 21, 28, and 35 dai, P. brassicae biomass in the susceptible cultivar became greater than in the resistant cultivar, which reflected the fact that, after 21 dai, resting spores started to form in the susceptible cultivar.

In planta expression of $12 P$. brassicae genes. The relative expression of $12 P$. brassicae genes over the time course in the resistant and susceptible cultivars was investigated using RT-qPCR. In the resistant cultivar, a single expression peak was observed for all 12 genes at 5 or 7 dai (Fig. 2), indicating the involvement of those genes in primary infection, production of secondary zoospores, or early secondary infection in the resistant cultivar. In the susceptible cultivar, the 12 genes could be classified into three groups according their expression patterns: G75 and G116 had an expression peak at 14 dai; G60, G117, and G10 had two expression peaks, at 14 and 35 dai; while the remaining genes had an expression peak at 35 dai.

Among the 12 genes, G116 and G117 have been reported to be upregulated in secondary plasmodia and secondary zoospores compared with primary zoospores (Feng et al. 2013a). All of the other genes were identified from a suppression subtractive hybridization cDNA library constructed from infected Arabidopsis plants at 30 dai (Bulman et al. 2006, 2007). This was consistent with the results of the current study: all of these genes, except G75 and G116, showed an expression peak at 35 dai in the susceptible cultivar (Fig. 2), indicating that they are important during the process of resting spore formation. The expression of all 12 genes in the resistant cultivar was downregulated after 7 dai, suggesting a restriction on their expression as a result of the resistance mechanism.

The genes specifically upregulated at 5 or 7 dai in the resistant cultivar may participate in the early resistance interaction and likely contribute to the ability of the pathogen to cause primary infections in the resistant cultivar, resulting in a level of primary infection similar to that observed in the susceptible cultivar. If the molecular interaction between canola and $P$. brassicae follows the traditional gene-forgene model (Feng et al. 2014), efforts to identify virulence genes should be focused on the candidates that have an expression pattern similar to these 12 genes.

Because the function of the 12 genes has not been studied, it is difficult to draw conclusions regarding their importance in pathogenicity, even if they were up- or downregulated during pathogenesis. Nonetheless, putative functions based on BLASTx suggested that some genes may play a role similar to their homologs. Among them are G10, G74, and G100, which showed a distinct upregulation in the resistant cultivar at 5 dai. G10 codes for a putative polysaccharide deacetylase, an enzyme that is known to deacetylate xylan and chitin. Primary infection of $P$. brassicae requires the action of plant cellwall-modifying enzymes to help the spores penetrate the cell wall. G74 codes for a putative glutathione S-transferase. In plants and many other organisms, this enzyme plays a role in chemical stress tolerance by conjugating and detoxifying toxic compounds (Neuefeind et al. 1997). Two genes encoding this enzyme from the plant pathogens Botrytis cinerea (Prins et al. 2000) and Alternaria brassicicola (Sellam et al. 2006) have been studied, and their in planta upregulation suggested their importance in fungal pathogenicity. In the current study, G74 was upregulated at 5 dai in the resistant cultivar, at which time its level of expression was 170 times higher than the Actin1 gene, suggesting that compounds toxic to $P$. brassicae may be involved in the resistance mechanism of the resistant cultivar. 
G100 was upregulated in primary zoospores but downregulated in secondary zoospores (Feng et al. 2013a). This gene codes for a putative cyclophilin A. Cyclophilins are a conserved family of proteins present in bacteria, fungi, plants, and animals, and are best known for being the cellular target of the immunosuppressive drug cyclosporin A (Marks 1996). Cyclophilin genes have been demonstrated to be virulence factors for both the plant fungal pathogen Magnaporthe grisea (Viaud et al. 2002) and the human fungal pathogen Cryptococcus neoformans (Wang et al. 2001). In M. grisea, the cyclophilin gene CYP1 influences the development of asexual reproductive structures and cellular turgor generation in appressoria (Viaud et al. 2002). The primary infection stages include zoospore formation and their release from the resting spores, which is analogous to the abovementioned stages of $M$. grisea. The specifically high expression of G100 at the primary infection stage suggests that this gene may have a function in $P$. brassicae similar to that of $C Y P 1$ in $M$. grisea.

The gene expression study indicated that the 12 genes were upregulated during the early infection stages in the resistant cultivar but at later infection stages in the susceptible cultivar. On the other hand, the microscopy investigation of infection indicated that the resistance is expressed phenotypically after secondary infection. The microscopy investigation was repeated with alternative seedling and inoculum preparations and, from one of the two repeats, plant samples

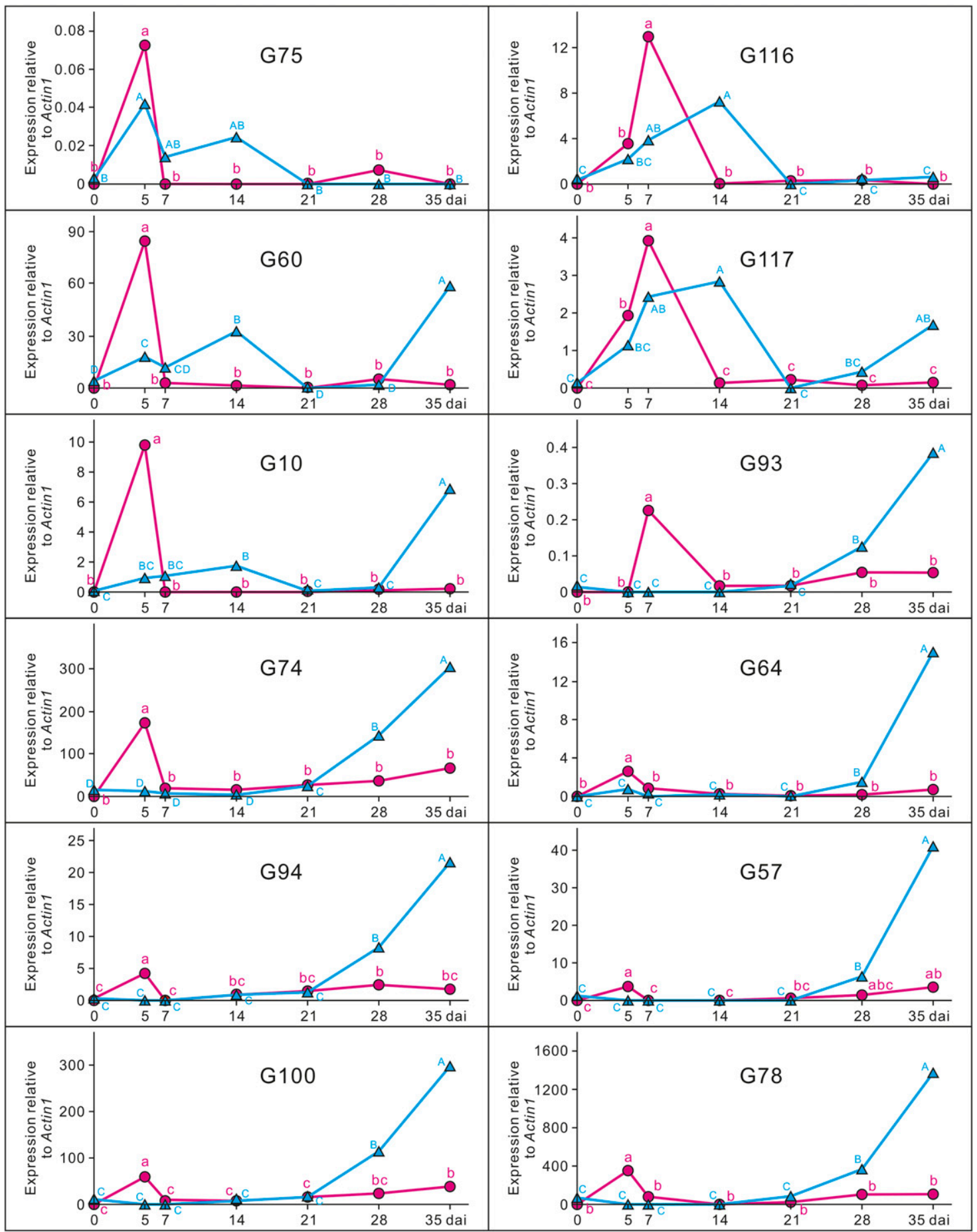

Fig. 2. Relative expression of 12 Plasmodiophora brassicae genes during the time course of infection in susceptible $45 \mathrm{H} 26$ canola (triangles) and resistant $45 \mathrm{H} 29$ canola (circles). Data points followed by the same letter do not differ based on Fisher's least significant difference at $P \leq 0.01$. 
were collected for RT-qPCR analyses of the gene expression. Similar results were obtained from the two microscopy investigations. In qPCR analysis, the three biological replications ruled out any possible technical errors during inoculation or nucleic acid manipulations. A reasonable biological explanation for our seemingly contradictory results is that the resistant cultivar does have a mechanism to resist primary infection but the pathogen overcomes this resistance by conscripting and elevating the expression of pathogenicity genes, and that this intensified host-pathogen interaction during the primary infection triggers further resistance observed at the stage of secondary infection.

\section{Acknowledgments}

Financial support for this study was received from the State Administration of Foreign Experts Affairs, P. R. China, the Alberta Crop Industry Development Fund, Western Grains Research Foundation, Canola Agronomic Research Program, and the Canola Science Cluster (GF2).

\section{Literature Cited}

Buczacki, S. T., and Clay, C. M. 1984. Some observations on secondary zoospore development in Plasmodiophora brassicae. Trans. Br. Mycol. Soc. 82: 339-342.

Bulman, S., Ridgway, H. J., Eady, C., and Conner, A. J. 2007. Intron-rich gene structure in the intracellular plant parasite Plasmodiophora brassicae. Protist 158:423-433.

Bulman, S., Siemens, J., Ridgway, H. J., Eady, C., and Conner, A. J. 2006. Identification of genes from the obligate intracellular plant pathogen, Plasmodiophora brassicae. FEMS Microbiol. Lett. 264:198-204.

Deora, A., Gossen, B. D., and McDonald, M. R. 2012. Infection and development of Plasmodiophora brassicae in resistant and susceptible canola cultivars. Can. J. Plant Pathol. 34:239-247.

Deora, A., Gossen, B. D., and McDonald, M. R. 2013. Cytology of infection, development and expression of resistance to Plasmodiophora brassicae in canola. Ann. Appl. Biol. 163:56-71.

Feng, J., Cao, T., Rennie, D. C., Strelkov, S. E., and Hwang, S. F. 2014. Hostparasite interactions in clubroot of crucifers. Can. J. Plant Pathol. 36 (Suppl. 1):113-321.

Feng, J., Hwang, R., Hwang, S. F., Strelkov, S. E., Gossen, B. D., Zhou, Q. X., and Peng, G. 2010. Molecular characterization of a serine protease Pro1 from Plasmodiophora brassicae that stimulates resting spore germination. Mol. Plant Pathol. 11:503-512

Feng, J., Hwang, S. F., and Strelkov, S. E. 2013a. Assessment of gene expression profiles in primary and secondary zoospores of Plasmodiophora brassicae by dot blot and real-time PCR. Microbiol. Res. 168:518-524.

Feng, J., Hwang, S. F., and Strelkov, S. E. 2013b. Studies into primary and secondary infection processes by Plasmodiophora brassicae on canola. Plant Pathol. 62:177-183.

Feng, J., Xiao, Q., Hwang, S. F., Strelkov, S. E., and Gossen, B. D. 2012. Infection of canola by secondary zoospores of Plasmodiophora brassicae produced on a nonhost. Eur. J. Plant Pathol. 132:309-315.

Gludovacz, T. V., Deora, A., McDonald, M. R., and Gossen, B. D. 2014. Cortical colonization by Plasmodiophora brassicae in susceptible and resistant cabbage cultivars. Eur. J. Plant Pathol. 140:859-862.
Hwang, S. F., Ahmed, H. U., Zhou, Q., Rashid, A., Strelkov, S. E., Gossen, B. D., Peng, G., and Turnbull, G. D. 2013. Effect of susceptible and resistant canola plants on Plasmodiophora brassicae resting spore populations in the soil. Plant Pathol. 62:404-412.

Hwang, S. F., Howard, R. J., Strelkov, S. E., Gossen, B. D., and Peng, G., 2014 Management of clubroot (Plasmodiophora brassciae) on canola (Brassica napus) in western Canada. Can. J. Plant Pathol. 36 (Suppl. 1):49-65.

Hwang, S. F., Strelkov, S. E., Feng, J., Gossen, B. D., and Howard, R. J. 2012 Plasmodiophora brassicae: A review of an emerging pathogen of the Canadian canola (Brassica napus) crop. Mol. Plant Pathol. 13:105-113.

Ingram, D. S., and Tommerup, I. C. 1972. The life history of Plasmodiophora brassicae Woron. Proc. R. Soc. Lond. Biol. 180:103-112.

Kageyama, K., and Asano, T. 2009. Life cycle of Plasmodiophora brassicae. J. Plant Growth Regul. 28:203-211.

Marks, A. R. 1996. Cellular functions of immunophilins. Physiol. Rev. 76: 631-649.

Naiki, T., and Dixon, G. R. 1987. The effects of chemicals on developmental stages of Plasmodiophora brassicae (clubroot). Plant Pathol. 36:316-327.

Neuefeind, T., Reinemer, P., and Bieseler, B. 1997. Plant glutathione S-transferases and herbicide detoxification. Biol. Chem. 378:199-205.

Peng, G., Lahlali, R., Hwang, S. F., Pageau, D., Hynes, R. K., McDonald, M. R. Gossen, B. D., and Strelkov, S. E. 2014. Crop rotation, cultivar resistance, and fungicides/biofungicides for managing clubroot (Plasmodiophora brassicae) on canola. Can. J. Plant Pathol. 36 (Suppl. 1):99-112.

Prins, T. W., Wagemakers, L., Schouten, A., and Van Kan, J. A. 2000. Cloning and characterization of a glutathione S-transferase homologue from the plant pathogenic fungus Botrytis cinerea. Mol. Plant Pathol. 1:169-178.

Rahman, H., Peng, G., Yu, F., Falk, K. C., Kulkarni, M., and Selvaraj, G. 2014 Genetics and breeding for clubroot resistance in Canadian spring canola (Brassica napus L.). Can. J. Plant Pathol. 36 (Suppl. 1):122-134.

Schmittgen, T. D., and Livak, K. J. 2008. Analyzing real-time PCR data by the comparative $C_{\mathrm{T}}$ method. Nat. Protoc. 3:1101-1108.

Schwelm, A., Fogelqvist, J., Knaust, A., Jülke, S., Lilja, T., Bonilla-Rosso, G., Karlsson, M., Shevchenko, A., Dhandapani, V., Choi, S., Kim, H., Park, J., Lim, Y., Ludwig-Müller, J., and Dixelius, C. 2015. The Plasmodiophora brassicae genome reveals insights in its life cycle and ancestry of chitin synthases. Sci. Rep. 5:11153.

Sellam, A., Poupard, P., and Simoneau, P. 2006. Molecular cloning of AbGst encoding a glutathione transferase differentially expressed during exposure of Alternaria brassicicola to isothiocyanates. FEMS Microbiol. Lett. 258: 241-249.

Strelkov, S. E., Tewari, J. P., Smith, E., and Smith-Degenhardt, E. 2006 Characterization of Plasmodiophora brassicae populations from Alberta, Canada. Can. J. Plant Pathol. 28:467-474.

Viaud, M. C., Balhadère, P. V., and Talbot, N. J. 2002. A Magnaporthe grisea cyclophilin acts as a virulence determinant during plant infection. Plant Cell 14:917-930.

Wang, P., Cardenas, M. E., Cox, G. M., Perfect, J. R., and Heitman, J. 2001. Two cyclophilin A homologs with shared and divergent functions important for growth and virulence of Cryptococcus neoformans. EMBO Rep. 2:511-518.

Xue, S., Cao, T., Howard, R. J., Hwang, S. F., and Strelkov, S. E. 2008. Isolation and variation in virulence of single-spore isolates of Plasmodiophora brassicae from Canada. Plant Dis. 92:456-462.

Zhang, H., Feng, J., Manolii, V. P., Strelkov, S. E., and Hwang, S. F. 2015. Characterization of a gene identified in pathotype 5 of the clubroot pathogen Plasmodiophora brassicae. Phytopathology 105:764-770. 